\title{
The influence of three different intercalation techniques on the microstructure of exfoliated graphite
}

\author{
Xandra van Heerden, Heinrich Badenhorst ${ }^{*}$
}

SARChI Chair in Carbon Materials and Technology, Department of Chemical Engineering, University of Pretoria, Lynnwood Road, Pretoria, 0002, South Africa

\begin{abstract}
The characteristics of exfoliated graphite derived from three intercalation methods: gas phase intercalation of iron (III) chloride, modified Hummers method and an electrochemical technique, were compared.

Despite the absence of strong oxidizers the electrochemical method produced a material which is very similar to that of the modified Hummers method in virtually every respect. These both produced a graphite oxide based material whilst the gas phase method resulted in a stage 1 intercalation compound. The different materials demonstrated very distinctive exfoliation behaviour.

The gas phase material exhibits 3\% mass loss during expansion but has a large amount of residual intercalant. The graphite oxide based methods result in mass loss of up to $25 \%$ in the expansion zone. For all three samples the residual impurities lead to a reduction in oxidative resistance. Once removed all samples exhibit nearly identical oxidation behaviour.

All three methods delivered graphite nanoplatelets with a very high aspect ratio through considerable expansion. Surprisingly the gas phase method caused persistent residual damage. All three methods yielded a product with varying levels of basal and edge damage, but the purified Hummers material exhibited marginally more 'ideal' characteristics. The simplest but still effective technique was found to be the electrochemical approach.
\end{abstract}

\footnotetext{
* Corresponding author. Tel: +27 12420 4989. Fax: +27 12420 2516. E-mail: carbon@up.ac.za
} 


\section{Introduction}

Graphite has a layered structure, which allows the introduction of various molecules or atoms between these layers, producing a material known as graphite intercalated compounds (GICs). There are various methods of intercalation, such as different liquid intercalation methods, gas phase intercalation, hydrothermal and electrochemical intercalation methods $[1,2]$.

GICs have many direct applications because of their unique properties. They can be used as additives, electrodes, conductors, etc. [3,4]. However, GICs can be expanded to produce a low density, large surface area, worm-like structure known as expanded graphite. Expansion can be achieved by using different methods such as microwave irradiation [5] and rapid heating, also known as thermal shock [6-8]. These expanded worms can in turn be broken down into nanoplatelets using for example sonication $[9,10]$.

These graphite nanoplatelets (GNPs) generally have a thickness of between 3 to $10 \mathrm{~nm}$ and a width ranging from a few hundred nanometers up to a few hundred micrometers. Under different conditions it is possible to further reduce the size of these structures down to a single graphene layer. GNPs are mainly used as an additive to improve mechanical properties and to enhance thermal or electrical conductivity [11-14]. Specifically, this material is increasingly being used to enhance the thermal conductivity of thermal energy storage media $[15,16]$ such as phase change materials (PCMs). In general the thermal conductivities of most PCMs are very low, leading to problems charging and discharging the storage units. By adding GNPs the conductivity can be significantly improved even at low loadings, leading to enhanced thermal performance. These applications cover both high and low temperature storage media, thus the oxidative stability of the additive is an important factor in determining service life. In addition, particles with a specific microstructure are sought, i.e. high aspect ratio, low structural deformation, intact crystal configuration for fast phonon propagation, low contaminant levels, etc.

The aim of this work is to develop a cost effective production method for GNPs suitable for use in PCM composites. As mentioned before, a myriad of techniques to produce these materials exist, each with varying costs and yields. However it is unclear how the different intercalation techniques affect the expansion behaviour, microstructure and related characteristics of the GNPs. True graphite intercalation 
compounds possess guest molecules inserted between the graphene layers [17]. It is assumed that the graphene layers in these complex materials remain largely intact with the guest molecules or atoms located in between graphene layers. The actual composition varies on a layer by layer basis as not every layer may be intercalated.

However, many techniques used to produce GICs actually create an intermediary known as graphite oxide (GO). GO is a highly oxidized and disordered form of graphite that contains carbon, oxygen, and hydrogen in variable ratios [18-20]. It is synthesized by exposing flake graphite to concentrated acids in conjunction with strong oxidizers, for example nitric acid and $\mathrm{KMnO}_{4}[18,20]$. Mermoux and Chabre [21] contend that GO is obtained via hydrolysis of an intermediary compound formed through over-oxidation of a GIC. Graphite oxide retains the lamellar structure of the parent graphite but with a much larger and irregular basal spacing $\left(\mathrm{d}_{002}\right.$ between 0.562 $\mathrm{nm}$ and $0.902 \mathrm{~nm}[22]$ ) owing to the presence of oxygen-containing groups and water. The nature of GO is not well defined but it is believed that it is comprised of lactone, phenol, carbonyl, ether, etc. functional groups randomly attached to the graphene layers.

It seems likely that there would be a distinct difference between the GNPs obtained through these dissimilar approaches. The objective of this investigation is to determine the impact of three different intercalation methods on the microstructure and associated characteristics of the final GNP product. The first intercalation method chosen is gas phase intercalation of iron (III) chloride. This method is known to produce a simple binary intercalation compound with clear staging [23-25]. The second technique is a modified Hummers method [18], which is known to produce graphite oxide [26,27]. The final approach is an electrochemical method using an applied voltage and only sulphuric acid as electrolyte [28]. It was reasoned that in the absence of the strong oxidizing agents used in the Hummers technique, this method may incur less damage to the final GNPs.

A variety of experimental conditions were explored for each of the three techniques, however only the one giving the greatest level of expansion will be reported on here. The characteristics of the resulting samples were determined through multiple techniques including an extensive examination using scanning electron microscopy. The samples were exposed to a mild oxidation to reveal any underlying structural defects which may not be immediately visible but are prone to 
attack. To discern between intrinsic characteristics and the effects of impurities, a portion of the samples were first purified before oxidation. This approach provides valuable insights into the material characteristics and leads to a clear distinction between the different techniques.

\section{Experimental}

In all cases, high purity, large, natural graphite flakes (RFL $99.5 \mathrm{O}$ ), with a particle size of min. $90 \%>160 \mu \mathrm{m}$, obtained from Graphit Kropfmühl AG were used. This sample has a carbon purity of $>99.5 \%$. The other chemical reagents, iron (iii) chloride, potassium permanganate, sulphuric acid (95\%-99\%), nitric acid (65 $\%)$, hydrochloric acid (32\%) and distilled water were supplied by Merck Chemical (Pty) Ltd. As a reference, a portion of the original material was further purified (see section 2.4) and also lightly oxidized.

\subsection{Gas phase intercalation}

The method used to prepare the gas phase intercalation compound was derived from the method used by [29]. A sealed aluminium cylinder was employed as a pressure vessel. The intercalation reaction was carried out with $6 \mathrm{~g}$ of anhydrous iron (III) chloride mixed with $3 \mathrm{~g}$ of graphite. The mixture was first dried at $50{ }^{\circ} \mathrm{C}$ for $2 \mathrm{~h}$ in a convection oven and immediately transferred to the reaction cylinder. The cylinder was perfectly sealed with high temperature silicon sealant and heated to the reaction temperature of $300{ }^{\circ} \mathrm{C}$ for $25 \mathrm{~h}$ in a muffle furnace. In an attempt to remove any excess iron (III) chloride, the product mixture was soaked in hydrochloric acid for $2 \mathrm{~h}$, then filtered and washed with distilled water. Finally the sample was dried in a convection oven.

\subsection{Modified Hummers method}

The method and amounts used to prepare the liquid phase intercalation compound were similar to the method used by Nakajima and Matsuo [30]. The approach used in this study combines concentrated nitric and sulphuric acids with potassium permanganate, thus a mixture of the classical Hummers and the StaudenmaierHofmann-Hamdi methods. The mass of graphite and potassium permanganate were fixed at $15 \mathrm{~g}$ and $3 \mathrm{~g}$ respectively. These were placed in a beaker to which $15 \mathrm{ml}$ of 
nitric acid and then $45 \mathrm{ml}$ of sulphuric acid were added under continuous mechanical stirring for 10 minutes at the ambient temperature. The mixture was removed from the stirrer and left for 60 minutes after which it was soaked in distilled water for $2 \mathrm{~h}$. The soaked mixture was filtered and washed with distilled water to neutrality after which it was dried in a convection oven.

\subsection{Electrochemical intercalation}

The method used to prepare the electrochemical intercalation compound was adapted from the method used by [28]. The electrochemical cell comprised a plastic beaker, stainless steel electrodes, a porous fabric bag and the electrolyte was sulphuric acid. A galvanostat was used to apply a voltage to the electrodes. The setup is shown schematically in Figure 1.

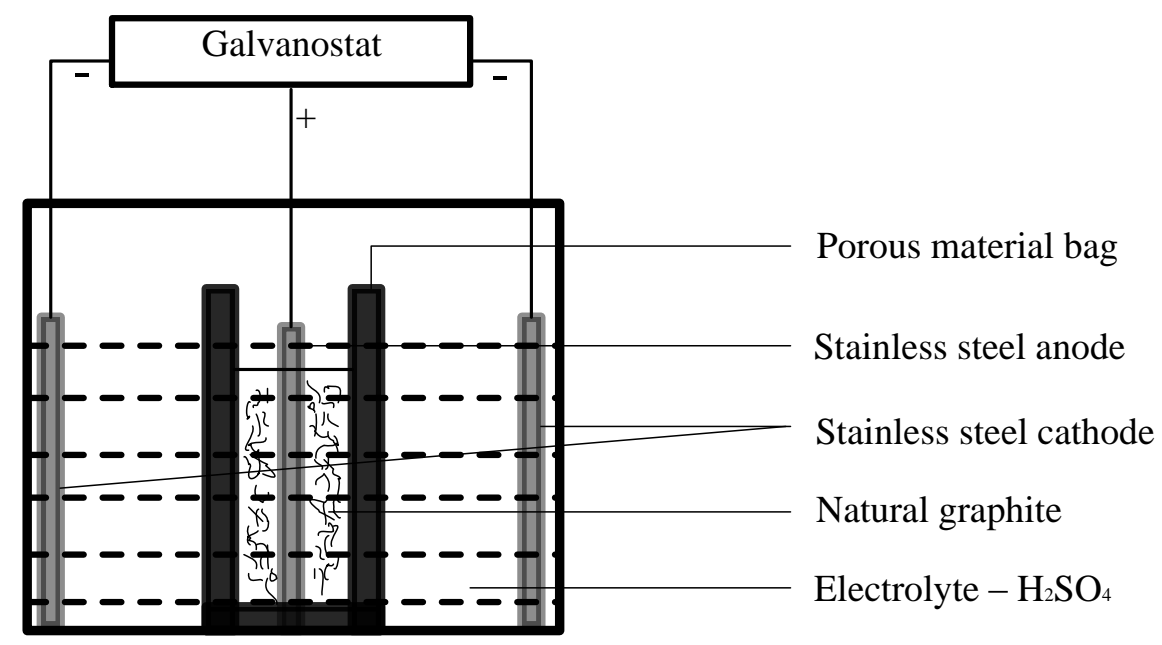

Figure 1: A schematic illustration of electrochemical intercalation method.

For the intercalation the bag was filled with graphite flakes. A stainless steel electrode was inserted in the middle of the bag to act as anode. The other stainless steel electrodes with the same size were placed parallel on either side of the bag. The cell was assembled and filled with the electrolyte. The electricity consumption was set at a constant at $10 \mathrm{~W}(2,5 \mathrm{~A}$ and $4 \mathrm{~V})$. After the reactor was left overnight, the GIC was filtered, washed to neutrality and then dried. 


\subsection{Expansion, sonication and purification}

Microwave irradiation was employed as the expansion method. All the samples produced from each intercalation method were expanded in a standard commercial microwave oven (1200W) for 2 minutes in a ceramic crucible.

The samples were then sonicated in order to produce the final GNP product. A mass of $300 \mathrm{mg}$ of each sample was submerged in $100 \mathrm{ml}$ of propan-2-ol. Under constant mechanical stirring, the samples were sonicated using a Sonics \& Materials VC 375 sonication probe ( 375 watt) for 10 minutes and left overnight to settle out after which they were dried in a rotary evaporator.

A portion of each sonicated sample was subjected to a very high temperature heat treatment at $2750{ }^{\circ} \mathrm{C}$ in a furnace (TTI model 1000-2560-FP20) under instrument grade Helium for $6 \mathrm{~h}$, followed by a very slow cool down. In the past this treatment has been found effective for removing even trace impurities present in graphite without damaging the sample [31], thus yielding an exceedingly pure material.

\subsection{Characterization}

Thermal characterization was conducted in a TA Instruments SDT Q600 thermogravimetric analyzer (TGA). Approximately 3-4 $\mathrm{mg}$ of each sample was placed in a $40 \mu \mathrm{l}$ alumina pan and subjected to three different treatments. Experiments were repeated multiple times and no discrepancies were observed.

A) Inert atmosphere

Intercalated samples were heated at a scan rate of $10^{\circ} \mathrm{C} \cdot \mathrm{min}^{-1}$ in instrument grade nitrogen flowing at $150 \mathrm{ml} \cdot \mathrm{min}^{-1}$ to a final temperature of $600{ }^{\circ} \mathrm{C}$. This measures the mass loss of the sample during expansion only. The final temperature was chosen based on the fact that the material would degrade significantly beyond this point under normal use in air.

B) Oxidizing atmosphere

Exfoliated samples (i.e. expanded and sonicated) were heated at a scan rate of $5{ }^{\circ} \mathrm{C} \cdot \mathrm{min}^{-1}$ in instrument grade oxygen flowing at $150 \mathrm{ml} \cdot \mathrm{min}^{-1}$ to a final temperature of $1000{ }^{\circ} \mathrm{C}$. This was repeated for both exfoliated and purified samples. This analysis gives an indication of the residual impurities present (i.e. ash content) since all the carbon will be removed by oxidation. In addition, the overall oxidative stability of the sample can be determined. 
C) Partial oxidation

In order to reveal the underlying microstructure samples were exposed to a mild oxidation. Samples were heated at a scan rate of $5^{\circ} \mathrm{C} \cdot \mathrm{min}^{-1}$ in instrument grade oxygen flowing at $150 \mathrm{ml} \cdot \mathrm{min}^{-1}$. Sample mass was continually monitored and when the mass loss reached around 5\%, the atmosphere was rapidly changed to inert and the temperature dropped to ambient to arrest oxidation.

Thermal expansion measurements were conducted on a TA Instruments TMA Q400 Thermo Mechanical Analyser (TMA). Just enough GIC flakes were placed in a platinum pan to cover the base of the pan (any more would lead to the sample expanding beyond the top edges of the pan). The flake expansion behaviour was measured with a flat-tipped standard expansion probe using an applied force of $0.2 \mathrm{~N}$. The temperature was scanned from $25^{\circ} \mathrm{C}$ to $600{ }^{\circ} \mathrm{C}$ at a scan rate of $10^{\circ} \mathrm{C} \cdot \mathrm{min}^{-1}$ in instrument grade nitrogen flowing at $50 \mathrm{ml} \cdot \mathrm{min}^{-1}$. Repeats were done for each experiment but no inconsistencies were found.

The powder X-ray diffraction (XRD) pattern of the graphite was obtained with 20 mass \% silicon as internal standard. A PANalytical X-pert Pro powder diffractometer with variable divergence and receiving slits coupled to an X'celerator detector using iron-filtered copper $\mathrm{K} \alpha$ radiation was used. The Raman spectra were acquired using a Dilor XY Raman spectrometer using the $\lambda=514.5 \mathrm{~nm}$ laser line of a coherent Innova 90 Ar+-laser. The samples were placed on a glass substrate, the laser intensity was 10 $\mathrm{mW}$ at the sample and no filters were used.

The sample surface area was measured using a Mircomeritics TriStar II surface area analyser by means of the 5 point BET method and nitrogen as adsorbent. An ARL Perform'X Sequential XRF (X-Ray Fluorescence) was used for the impurity analysis. Scanning electron microscope (SEM) images were obtained using an ultrahigh-resolution field-emission microscope (Zeiss Ultra Plus 55 FEGSEM) equipped with an in-lens detection system and operated at an acceleration voltage of $1 \mathrm{kV}$. A working distance of between 2 and $3 \mathrm{~mm}$ was used. The powder was lightly deposited on carbon tape and examined without any additional sample preparation. 


\section{Results}

\subsection{Intercalated and reference materials}

Before the different exfoliated graphite materials are examined, it is worthwhile to consider the intercalated compounds. Shown below in Figure 2 are the XRD diffractograms for all three intercalated samples, as a reference the purified natural graphite sample is included.

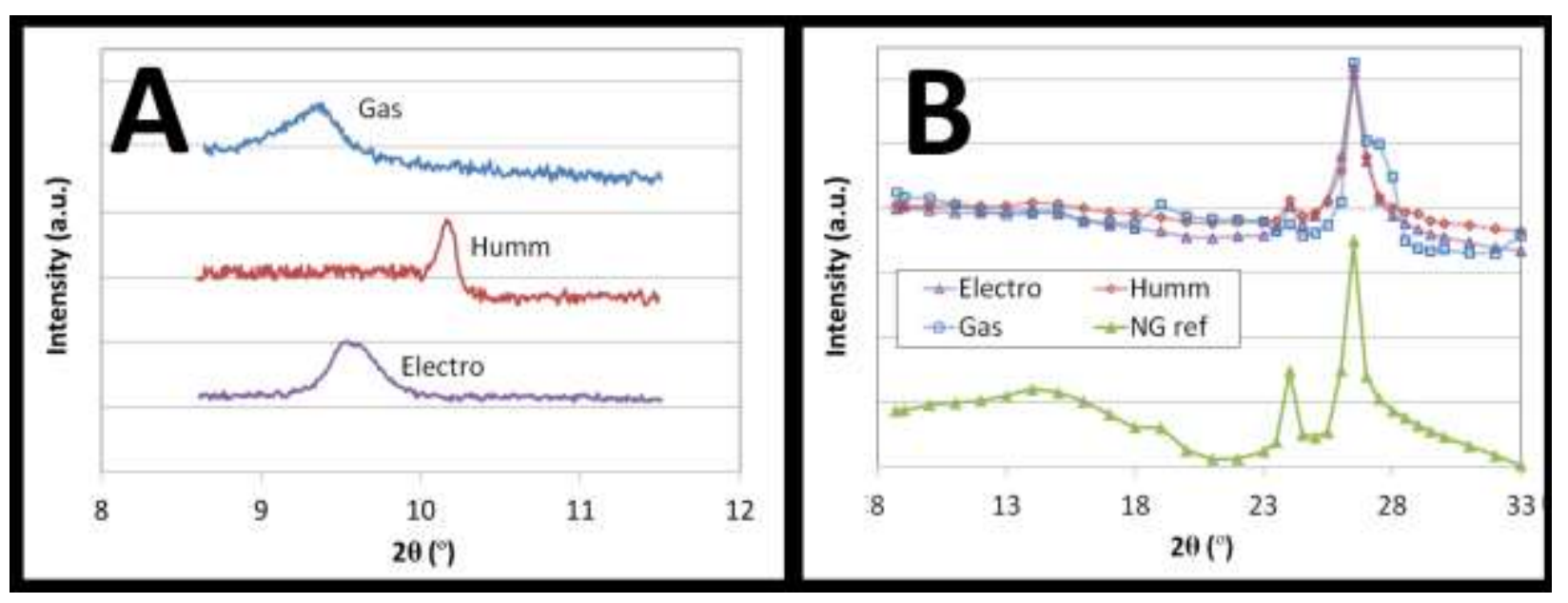

Fig. 2: XRD diffractograms of (A) intercalated and (B) exfoliated materials with natural graphite reference

The reference sample shows the characteristic 002 graphite peak at the expected value of $2 \theta=26.5^{\circ}$ as can be seen in Figure 2 (B). For the gas phase method a peak around $2 \theta=9.4^{\circ}$ can be seen in Figure $2(\mathrm{~A})$, which corresponds to an interlayer spacing of $0.94 \mathrm{~nm}$. This is in excellent agreement with literature values for a stage 1 intercalation compound [23, 32, 33]. The modified Hummers and electrochemical methods show similar behaviour with smaller interlayer spacings than the gas phase method. The values of $2 \theta$ around $10.2^{\circ}$ and $9.6^{\circ}$ indicate interlayer spacings of 0.87 and $0.92 \mathrm{~nm}$ for the modified Hummers and electrochemical methods respectively. These are in the range expected from literature for graphite oxide materials.

It can be seen in Figure 2 (B) that the standard 002 peak is recovered completely for all the exfoliated samples. However, the exfoliated gas phase sample retains a shoulder to the right of the main peak, most likely caused by a residual impurity. As will be seen later this sample does in fact contain a significant amount of iron based impurity. In all cases the pristine graphite inter-layer spacing is restored upon 
exfoliation. Due to the lack of $\mathrm{CuK}_{\beta}$ filtering a secondary peak is noticeable in the reference at $2 \theta=23.9^{\circ}$ and this peak too is recovered for all three exfoliated samples.

Thus the XRD results indicate that once the expanded graphite has been exfoliated the graphitic structure should be fully restored in the graphite nanoplatelets. Thus a purified natural graphite reference material can provide valuable insights into the expected "ideal" characteristics for the final GNPs. The basal plane of the lightly oxidized pure natural graphite is displayed below in Figure 3 (A).

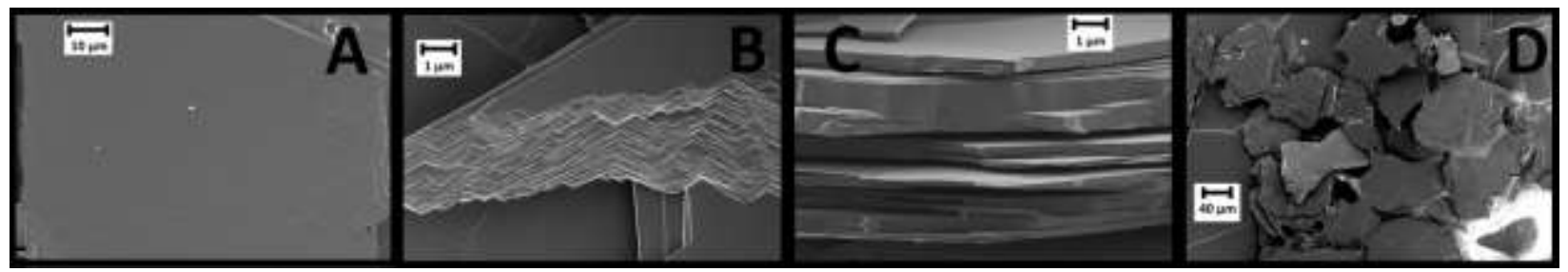

Fig. 3: Oxidized, pure natural graphite flakes

The surface exhibits the expected behaviour: due to the strong in plane covalent bonding attack by oxygen is limited to the edges. The basal surface remains very smooth without any visible damage. When the edges are examined more closely in Figure 3 (B), the characteristic edge structures can be seen. The edges are all comprised of impeccably straight lines, coupled with $60^{\circ}$ angles. These angles are the result of the underlying hexagonal crystal structure in the pristine natural graphite. A face-on view of the edge is given in Figure 3 (C). The edges exhibit the anticipated layered structure of graphite, but they are generally smooth, with continuous regions in excess of $1 \mu \mathrm{m}$ and overall flake thickness usually exceeds $30 \mu \mathrm{m}$. When the oxidized flakes are observed from a distance, as in Figure 3 (D), each individual flake is easily discernable. Flake boundaries are defined by clear, straight lines and very little agglomeration or inter-linking is observed.

\subsection{Gas phase intercalation}

As an initial indication of the extent of expansion, the density is often measured immediately after expansion. However, this approach can yield a highly variable result since expansion is random and chaotic leading to a highly disorganized structure. The bulk density, especially for low density, elongated particles such as these, depends heavily on the packing disorder. Simply measuring the post-expansion density may give an inaccurate value, so instead the tap density was chosen as a rough 
estimate of expansion effectiveness. The tap density is determined by exposing the sample to a repeatable compaction process in this case manual tapping.

An additional source of error is the presence of any impurities which add to the measured weight of the sample. Since the different methods may result in varying amounts of impurities incorporated through the intercalation process, the mass of the sample on an ash free basis should be used. For the gas phase sample the specific volume (inverse of tap density) was found to be $45.9 \mathrm{ml} . \mathrm{g}^{-1}$. In addition, the surface area for this sample was found to be around $14.1 \mathrm{~m}^{2} \cdot \mathrm{g}^{-1}$. For a more detailed analysis of the expansion behaviour the TMA result and the TGA mass loss curve under inert atmosphere are presented in Figure 4 (A).

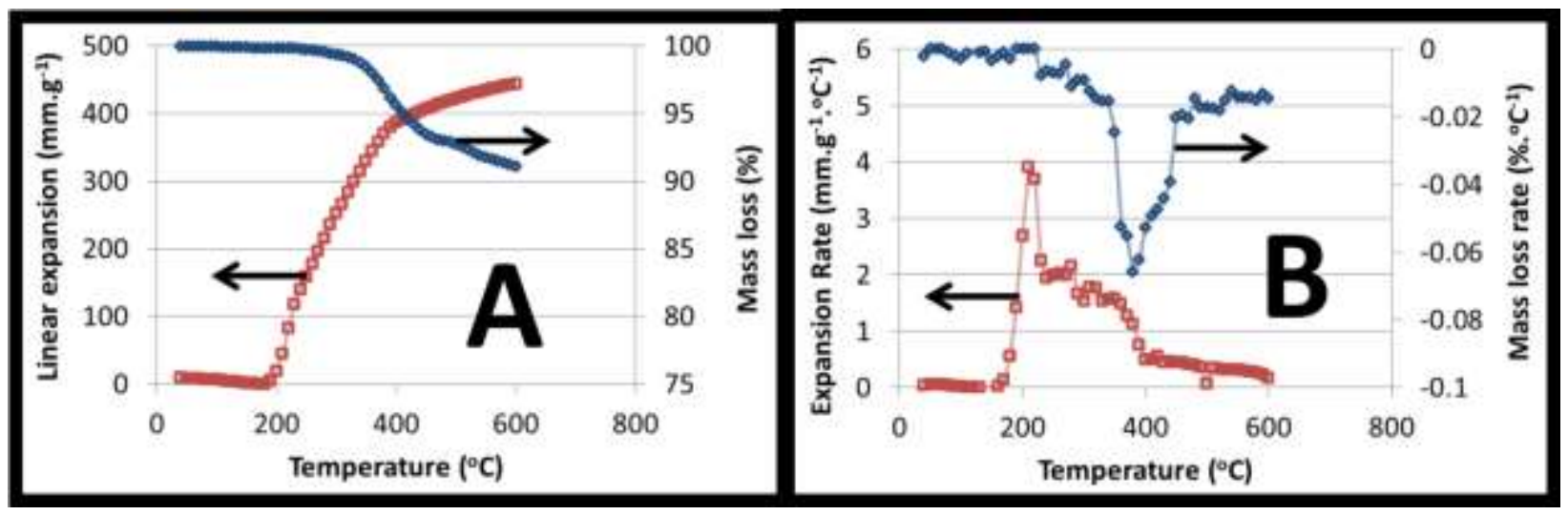

Fig. 4: Expansion, mass loss and rate curves for sample obtained with the gas phase intercalation method

Expansion occurs gradually from around $200{ }^{\circ} \mathrm{C}$ and is to a large extent completed at around $400{ }^{\circ} \mathrm{C}$, achieving a final maximum value of $440 \mathrm{~mm} \cdot \mathrm{g}^{-1}$. However, the majority of the mass loss for this sample only occurs at the final temperature. In fact $85 \%$ of the final expansion was achieved with a mass loss of less than $3 \%$. This mismatch is further evidenced when the expansion and mass loss rates in Figure 4 (B) are considered.

The expansion rate initially increases rapidly to $4 \mathrm{~mm} \cdot \mathrm{g}^{-1} \cdot{ }^{\circ} \mathrm{C}^{-1}$ before slowing and then maintaining a rate of around $2 \mathrm{~mm} \cdot \mathrm{g}^{-1} \cdot{ }^{\circ} \mathrm{C}^{-1}$ for the majority of the expansion. The mass loss rate on the other hand gradually increases from around $300{ }^{\circ} \mathrm{C}$, becoming more negative, before rapidly accelerating just before $400{ }^{\circ} \mathrm{C}$. The decomposition onset temperature for iron (III) chloride is around $330{ }^{\circ} \mathrm{C}$. Thus it is likely that if the sample contains large amounts of residual iron (III) chloride, this mass loss represents the decomposition of this material. Close examination of the 
exfoliated material in the SEM did in fact indicate large quantities of a non-graphitic contaminant, as demonstrated in Figure 5.

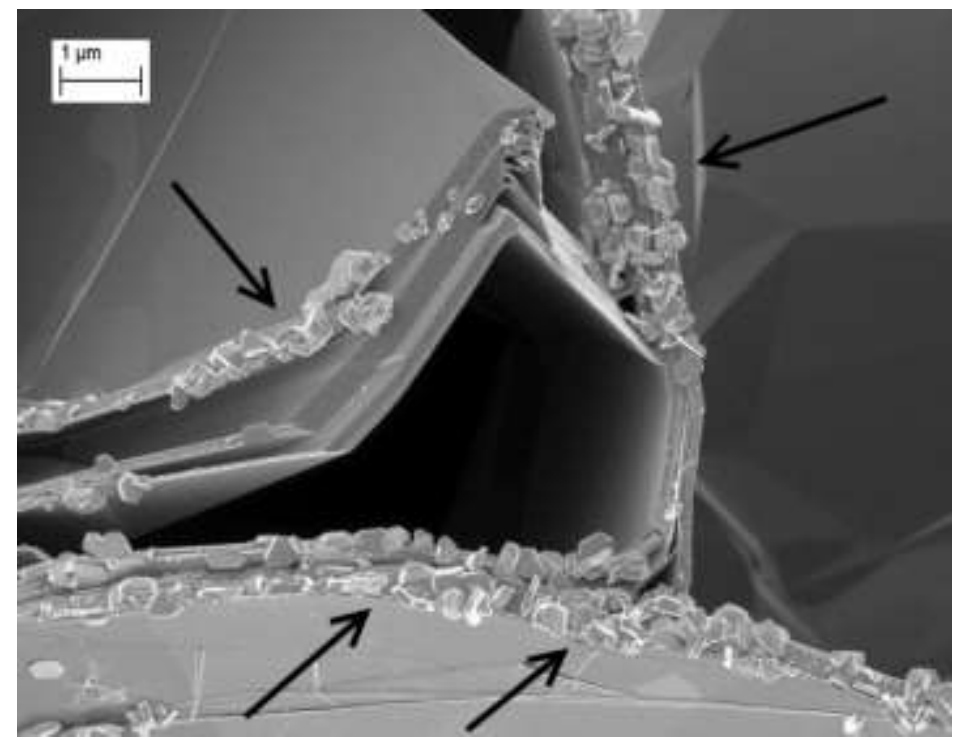

Fig. 5: Impurity found on exfoliated gas phase material

Curiously, the impurity appears to be preferentially concentrated at, or possibly even bound to, the graphite edges. Analysis of the sample was done using XRF which confirmed the presence of iron $(8 \mathrm{wt} \%)$ in the exfoliated graphite reaffirming the nature of the impurity as residual intercalant. It is clear that the hydrochloric acid soak was unsuccessful in removing the residual iron (III) chloride. To provide an indication of the impurity amount, the ash content of the exfoliated sample is found using oxidative TGA as given in in Figure 6.

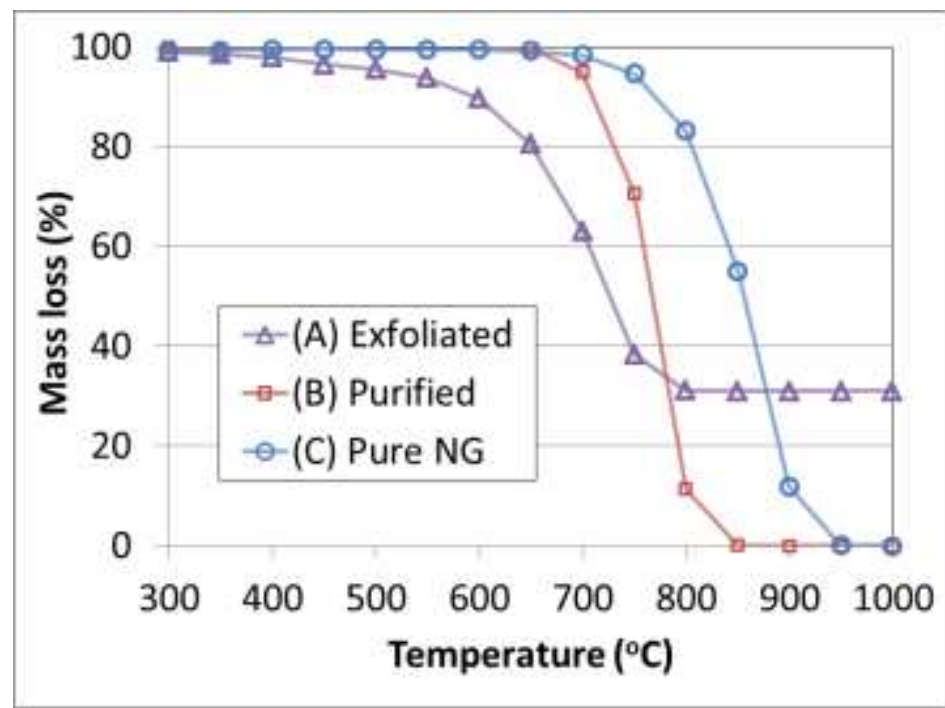

Fig. 6: Oxidative atmosphere TGA results for gas phase exfoliated (A) and purified (B) samples and pure natural graphite reference $(\mathrm{C})$ 
Thus the ash content is found to be $30 \%$ before purification. Since the iron (III) chloride decomposes and under oxidizing conditions most likely forms iron oxide, the true impurity mass will be somewhat higher. The presence of iron oxide is further confirmed by the red colour of the ash. Once purified this contaminant is completely removed and when the carbon is reacted away by oxidation the sample shows a residual mass of practically zero. Since graphite is very sensitive to especially metallic contaminants during oxidation it is not unexpected to find large amounts of damage when the exfoliated sample (A) is oxidized, as demonstrated in Figure 7.

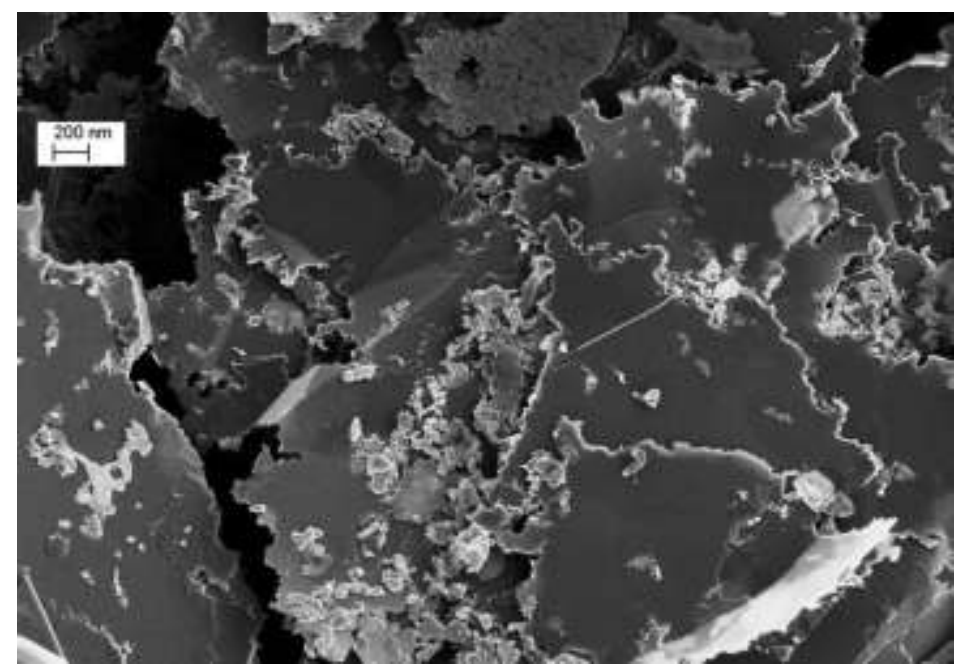

Fig. 7: Oxidized exfoliated gas phase sample

This image is typical of contaminated natural graphite [34], the edges are chaotic and ragged due the catalytic effect of the impurity. Consequently this material has a higher oxidative reactivity compared to the purified material (B). In contrast, it is anticipated that the purified sample will exhibit no such behaviour. Furthermore, as illustrated by the XRD, gas phase intercalation provided a true $1^{\text {st }}$ stage GIC. Meaning only insertion of the atoms/molecules between the graphene layers occurred and it is expected that no modification of the graphite crystalline structure has taken place. Thus one may assume that the "ideal" behaviour of the graphite would be restored. However this is not the case as can be seen from Figure 8. 


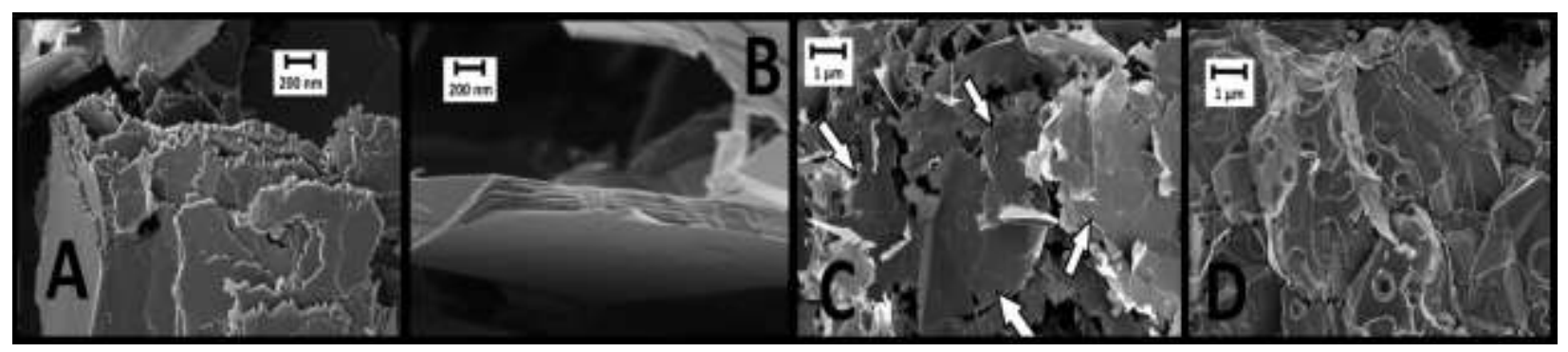

Fig. 8: Oxidized purified gas phase sample

The edges in Figure 8 (A) still remain highly chaotic and irregular, no distinct, straight lines are visible, perhaps indicating that some persistent modification to the structure has occurred. This behaviour is supported by the TGA result which shows that the purified material still has a higher reactivity than the pure parent material. To a certain extent this can be explained by the exfoliation process which leads to increased edge exposure and hence reactivity. Indeed it was found that in general the edge widths do not exceed a couple of hundred nanometers, as seen in Figure 8 (B).

This indicates that GNPs were indeed produced. Furthermore it is possible to find fairly large flakes where the basal region seems to be essentially intact, as demonstrated by Figure 8 (C). However, several cases were found where the basal surface itself is very heavily damaged, as shown for example in Figure 8 (D). In this case the surface is heavily cratered and contains several creases or bends. This seems to reinforce the idea that some persistent structural damage has taken place, since not a single case was found were the "ideal" behaviour was recovered.

\subsection{Modified Hummers method}

For this method the specific volume of the sample (on an ash free basis) was found to be $68.3 \mathrm{ml} . \mathrm{g}^{-1}$ and the surface area for this sample was measured at 21.7 $\mathrm{m}^{2} \cdot \mathrm{g}^{-1}$. The TMA result and the TGA mass loss curve under inert atmosphere are presented in Figure 9. 


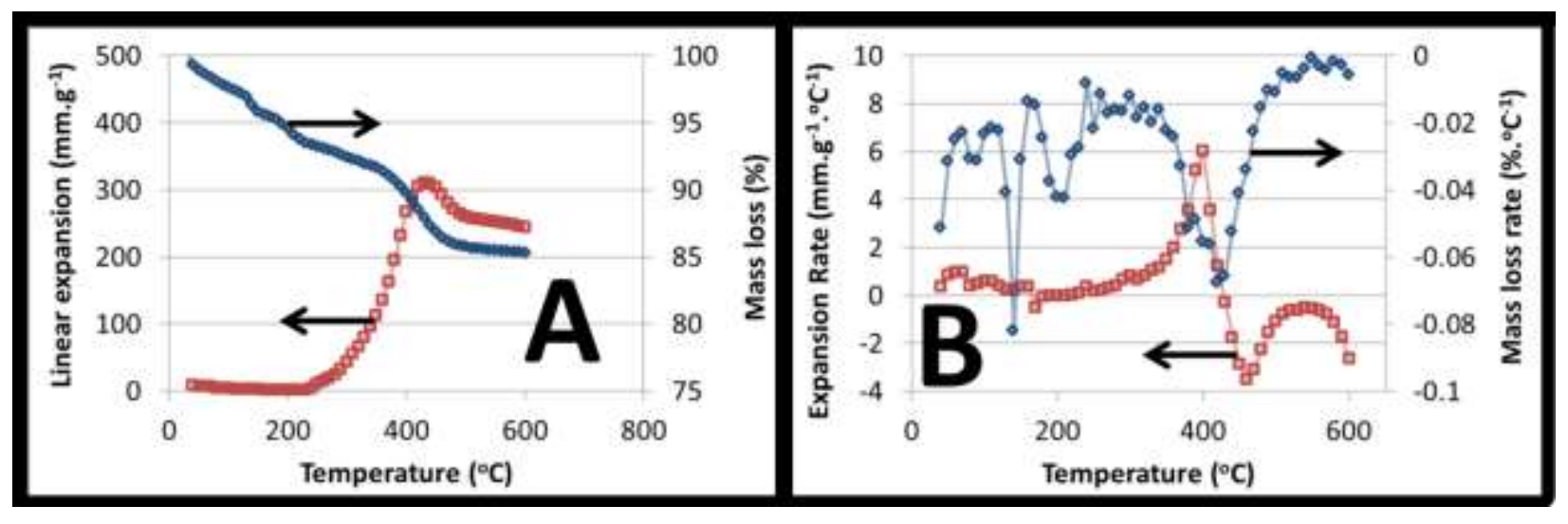

Fig. 9: Expansion, mass loss and rate curves for the sample obtained with the modified Hummers method

Expansion behaviour for this sample is quite different from the gas phase GIC. Starting slowly around $300{ }^{\circ} \mathrm{C}$ it rapidly accelerates as $400{ }^{\circ} \mathrm{C}$ is approached. After reaching a peak expansion around $420{ }^{\circ} \mathrm{C}$ the measured expansion decreases as the sample seemingly contracts. The mass loss curve is also quite different from the gas phase material, showing several discrete stages of mass loss. This effect is more readily observable from the expansion and mass loss rates curves given in Figure 9 (B).

The mass loss rate shows several peaks, most notably at 140, 200, 380 and $420{ }^{\circ} \mathrm{C}$. This behaviour is typical for the thermal desorption of surface bound oxygen complexes such as lactones, phenols, carbonyls, ethers, etc. [35]. Since it is expected that the Hummers method leads to the formation of graphite oxide, this behaviour is not unexpected. The mass loss around $380^{\circ} \mathrm{C}$ and to a lesser extent $420^{\circ} \mathrm{C}$, is directly associated with the rapid increase in the expansion rate up to $6 \mathrm{~mm} \cdot \mathrm{g}^{-1} \cdot{ }^{\circ} \mathrm{C}^{-1}$.

Graphite oxide is known to undergo explosive expansion during thermal treatment [36]. For the majority of the expansion, the expansion rate of the Hummers sample is nearly triple that of the gas phase material, reinforcing this notion. Both the specific volume and surface area of the Hummers method significantly exceed the values obtained for the gas phase compound. However, the final expansion measured by the TMA is around $244 \mathrm{~mm} \cdot \mathrm{g}^{-1}$, almost half the final value of the gas phase sample.

This seeming contradiction may be explained by considering the fact that expansion in the TMA occurs under an applied force. For the gas phase material, expansion occurs gradually, it has very little associated mass loss and hence gas generation. In contrast the graphite oxide material expands rapidly, releasing large 
amounts of gas and losing around $10 \%$ of its mass during this period. As this gas mixture expands and escapes, it leaves behind a highly porous and disrupted structure with regions where proper support is lacking. The resulting structure is shown below in Figure $10(\mathrm{~A})$.

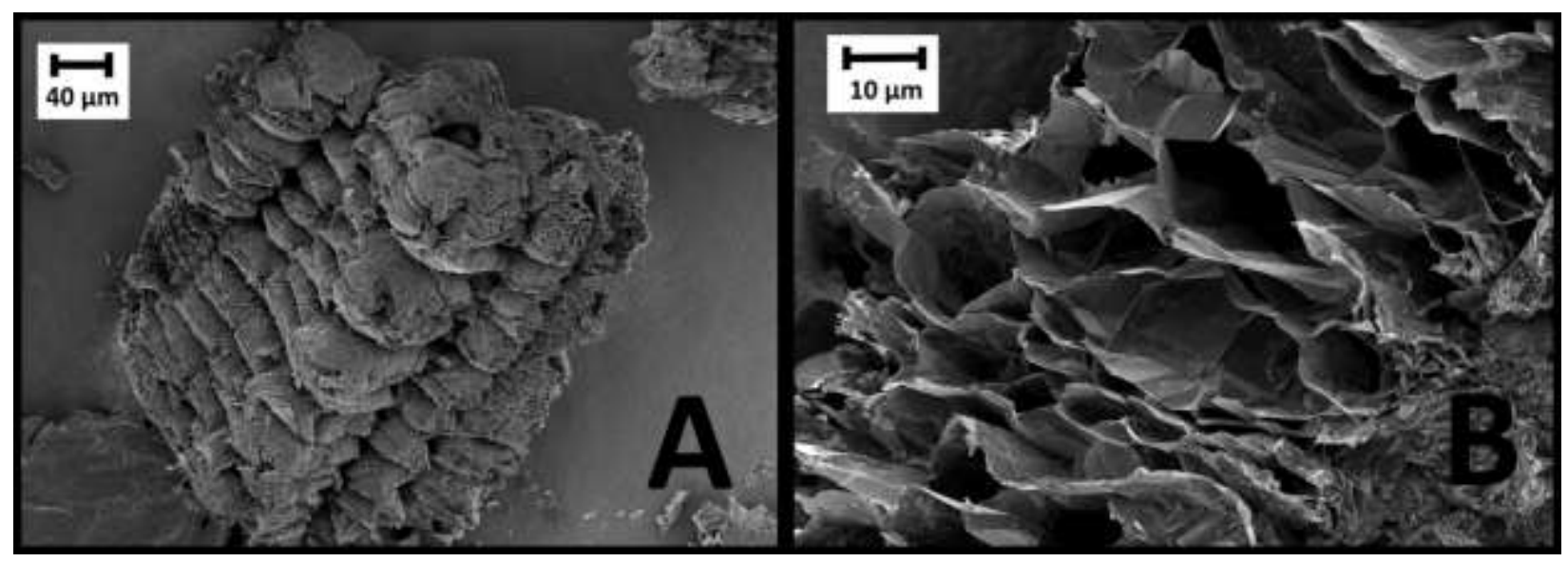

Fig. 10: Structure of expanded graphite samples obtained with the (A) Hummers and (B) gas phase methods

The Hummers method tends to create large puffed regions during exfoliation, resembling a concertina. The reason for this may be the segmented stacking structure found in this type of graphite [31]. The gas phase material on the other hand shows a more evenly distributed expansion, due the $1^{\text {st }}$ stage intercalation, rather resulting in a lattice work structure as exemplified in Figure 10 (B). Thus under the force of the TMA probe (which was set at its minimum value), the Hummers based material collapses once the gas starts to escape, registering a decrease in expansion and an incorrect estimate for the final expansion value. The Hummers method has a greatly reduced ash content of around 9\%, as can be seen from the TGA curves in Figure 11. 


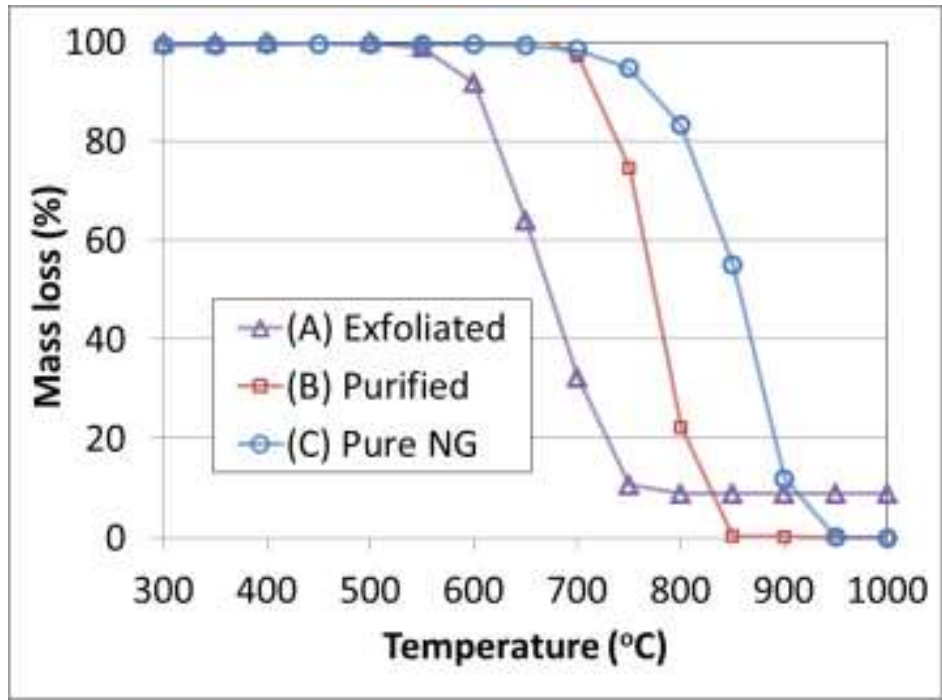

Fig. 11: Oxidative atmosphere TGA results for Hummers method exfoliated (A) and purified (B) samples and pure natural graphite reference $(\mathrm{C})$

In this case XRF analysis confirmed the presence of manganese $(2 \mathrm{wt} \%)$ in the exfoliated material, which is supported by the black ash colour. This is not unexpected since potassium permanganate was used as an oxidizer for this method. Whilst this material still exhibits the tell-tale signs of catalytic activity, the effect does not seem as pronounced as for the gas phase material, as demonstrated in Figure 12.

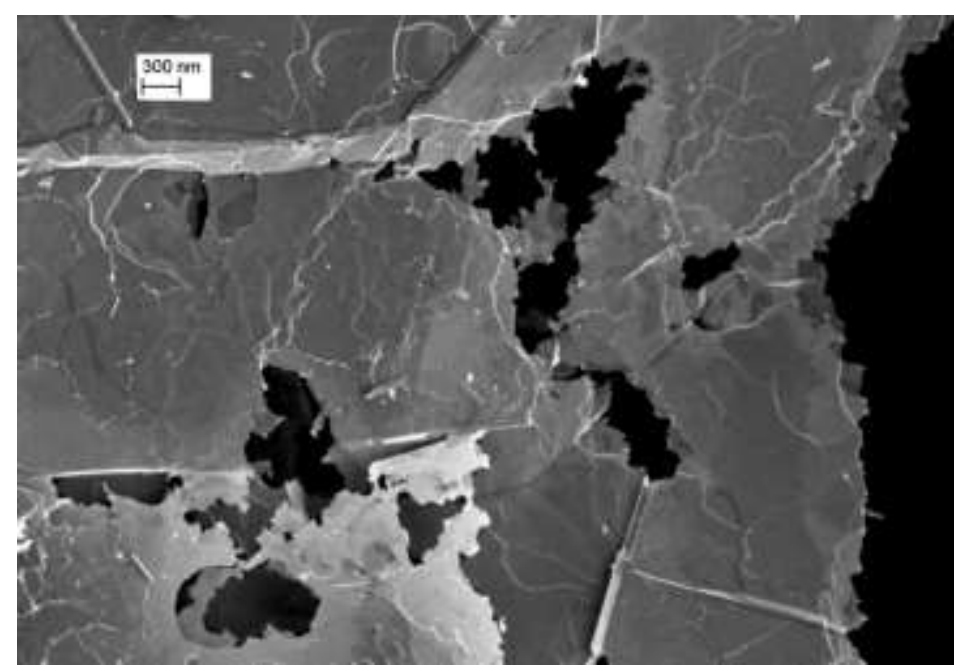

Fig. 12: Oxidized exfoliated Hummers sample

This may be due to the lower concentration or different species of catalyst present. Upon purification this residual impurity is fully removed, reducing the oxidative reactivity of the sample. However, as was the case for the gas phase material, the reactivity of the purified material still remains higher than that of the parent graphite. 
Nonetheless, for this material a significant change has taken place in the oxidation characteristics, as illustrated in Figure 13.

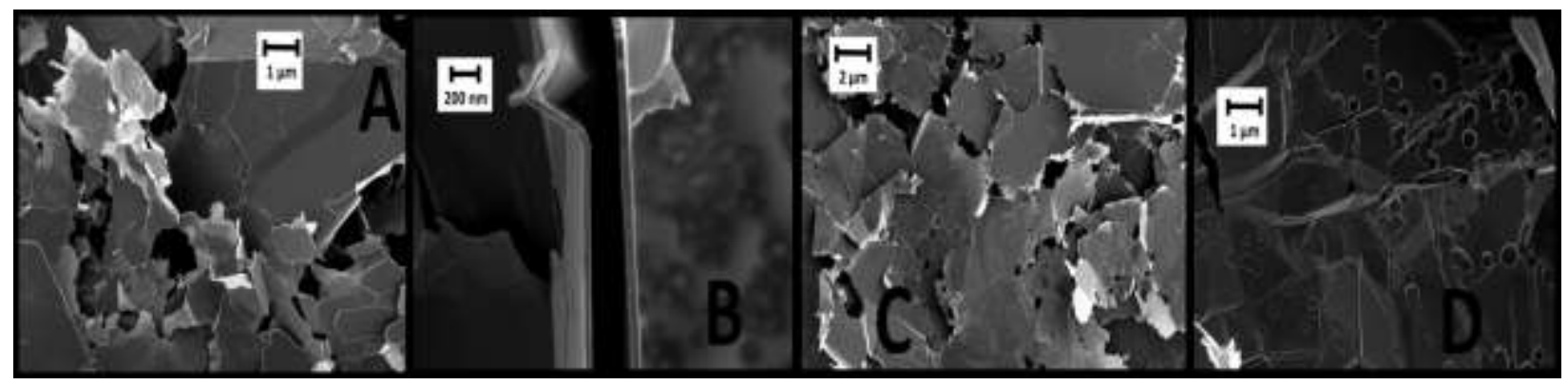

Fig. 13: Oxidized purified Hummers sample

While the archetypal $60^{\circ}$ angles have not been fully recovered, the edges do show significant proportions of straight line behaviour. As can be seen from this image, in general this technique has led to the degradation of the bulk particles into smaller agglomerated or interlinked platelets, which may account for the higher reactivity. Similarly to the other samples it was found that overall the edge widths for this material do not exceed a couple of hundred nanometers, as shown in Figure 13 (B).

This indicates that for this method graphite nanoplatelets have also been produced. In this case almost all flakes contain fairly large regions of intact and smooth basal surface area, as illustrated by Figure 13 (C). That being said, regions of moderate damage were also occasionally found, as shown for example in Figure 13 (D). The damage however seems to be mainly localized at the surface, without deep penetration into the sample. For example the pitting in the bottom left hand corner of Figure 13 (D) has almost disappeared, revealing the largely intact basal surface below.

\subsection{Electrochemical method}

For this technique the specific volume of the sample (on an ash free basis) was found to be $65.8 \mathrm{ml} \cdot \mathrm{g}^{-1}$ and the surface area for this sample was measured at 18.7 $\mathrm{m}^{2} \cdot \mathrm{g}^{-1}$, slightly less but close to the modified Hummers method values. The TMA and the TGA curves are given in Figure 14. 


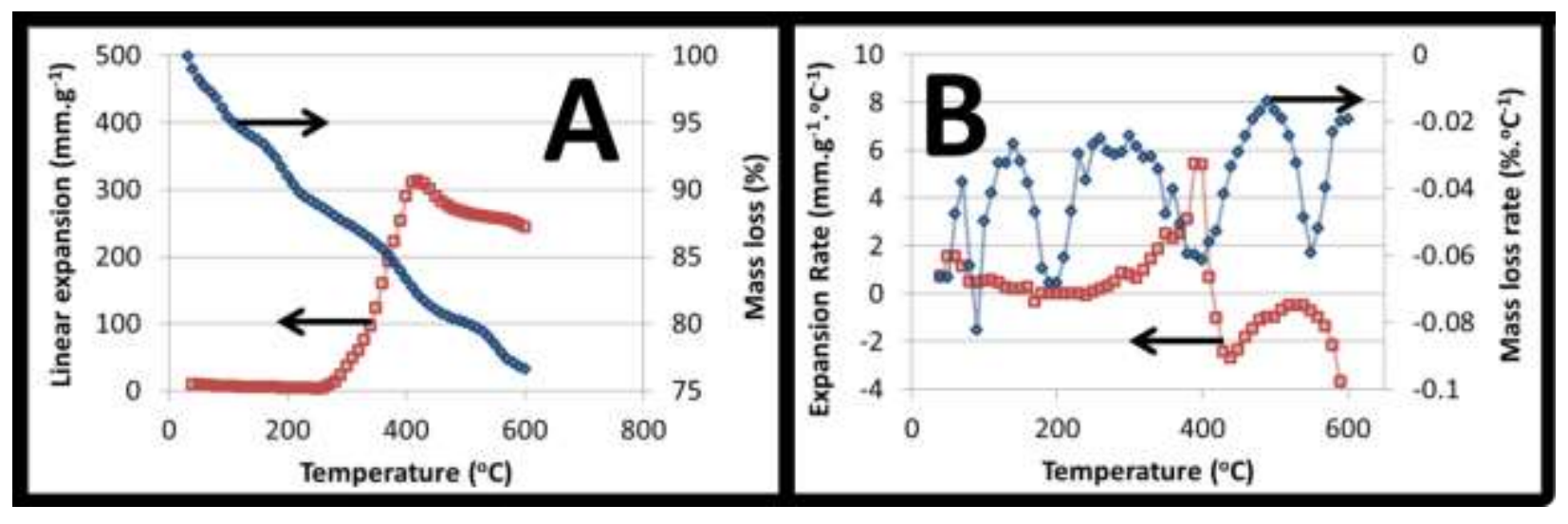

Fig. 14: Expansion, mass loss and rate curves for the sample obtained with the electrochemical method

Unexpectedly, the expansion and mass loss behaviours for this approach are remarkably similar to the Hummers method. Expansion occurs in an almost identical fashion with a final expansion of around $251 \mathrm{~mm} \cdot \mathrm{g}^{-1}$. Mass loss also occurs in steps at discrete temperatures, however in this case the mass loss after expansion is around $20 \%$, compared to the $15 \%$ found for the Hummers material. The expansion and mass loss rates curves given in Figure 14 (B) to provide additional insight. The expansion rate achieves virtually the same maximum value at just under $6 \mathrm{~mm} \cdot \mathrm{g}^{-1}$. ${ }^{\circ} \mathrm{C}^{-1}$. The mass loss rate has slightly different peak values of $90,200,380$ and $550{ }^{\circ} \mathrm{C}$. It is interesting to note that the expansion peak largely overlaps with the $380{ }^{\circ} \mathrm{C}$ mass loss peak, as was the case for the Hummers method. In addition, the largest portion of mass loss, again around $10 \%$, occurs in this region.

This evidence suggests that despite the fact that no oxidizers were present; the applied voltage was enough to induce graphite oxide formation in the presence of the sulphuric acid. It also implies that the same dominant surface complex is responsible for the expansion in both cases. In contrast to the Hummers method, this sample has a slightly increased ash content of around 16\%, as shown by the TGA curves in Figure 15. 


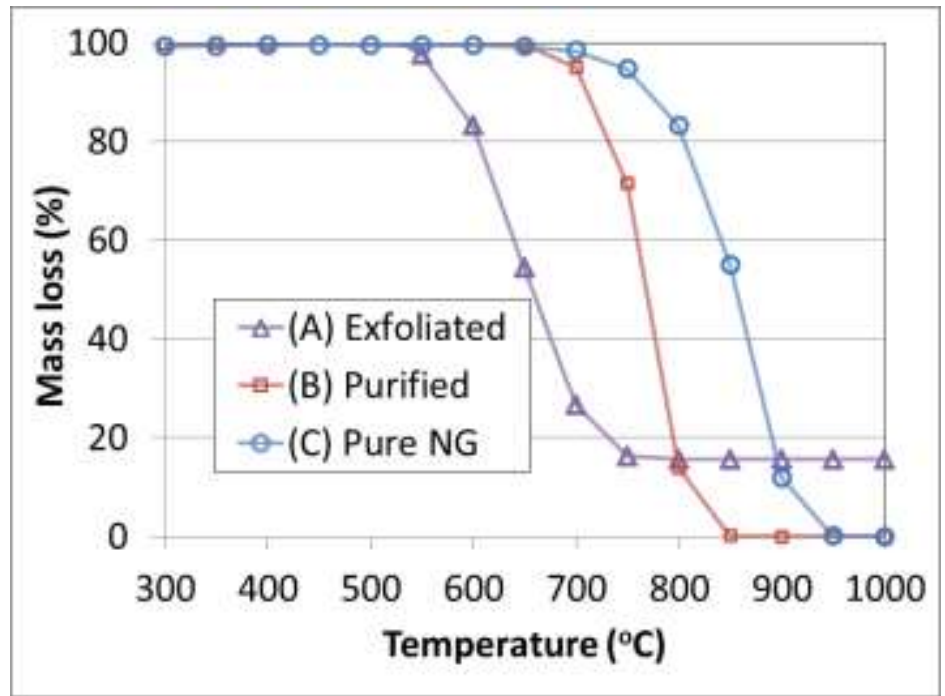

Fig. 15: Oxidative atmosphere TGA results for electrochemical method exfoliated (A) and purified (B) samples and pure natural graphite reference $(\mathrm{C})$

Notably in this case the XRF indicated the presence of iron $(0.5 \mathrm{wt} \%)$ in the exfoliated material, which was supported by the red ash colour. As only high purity sulphuric acid was used, it is likely that the acid leached some of the iron from the electrodes, which was subsequently incorporated into the GIC. However, in this case the catalyst activity is not quite as pronounced as for the gas phase material, as can be seen in Figure 16 (A).

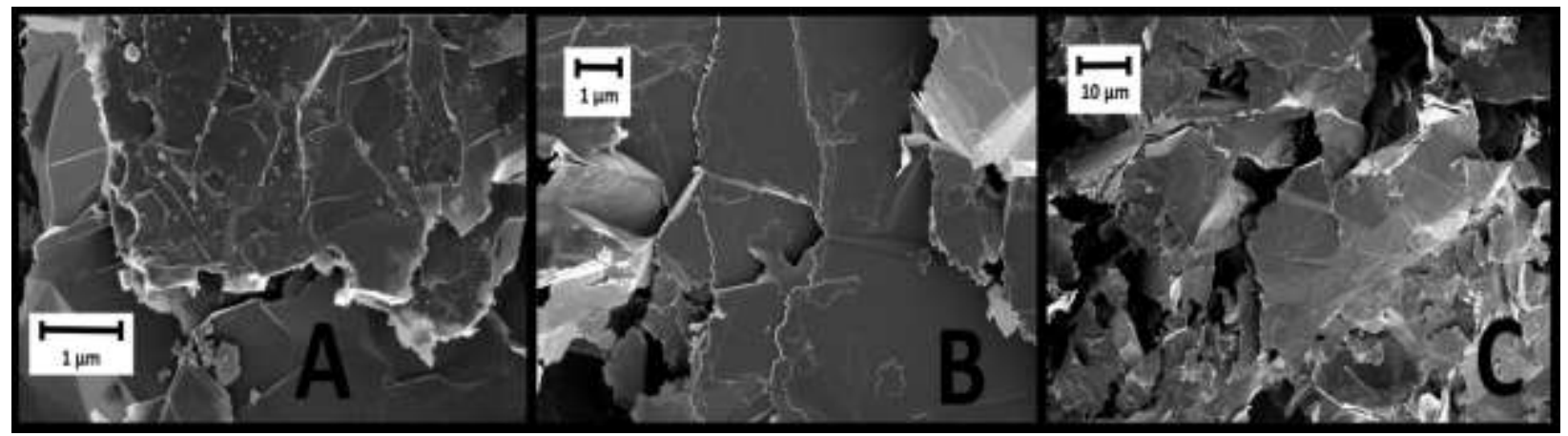

Fig. 16: Oxidized exfoliated electrochemical sample

The edges more closely resemble the Hummers material, possibly due to the fact that the iron is initially present probably as a sulphate rather than a chloride as was the case for the gas phase sample. As expected the purification step removes the impurity but again does not restore the reactivity to that of the parent graphite. Similarly to the other materials, the reactivity of the purified sample still remains higher than pristine 
graphite. The oxidation behaviour of the purified material is illustrated in Figure 16 (B).

In this case the behaviour more closely resembles the gas phase material and no straight-line edges are visible. Since it was commonly found that platelet edge thickness was less than a few hundred nanometers, this method also created graphite nanoplatelets. Similarly to the Hummers method almost all flakes contain large regions of intact and smooth basal surface area, as illustrated by Figure 16 (C). For this sample however, regions of concentrated damage such as pits or basal surface disruption were not readily found.

\subsection{Method comparison}

Based on the XRD results it is clear that two different intercalation phenomena were taking place. For the gas phase material a stage 1, pure intercalation compound was created. The other two intercalation methods do not exhibit clear staging behaviour but did indicate an increase in the interlayer spacing. The difference is further evidenced by the expansion behaviour observed in the TMA, shown comparatively in Figure 17.

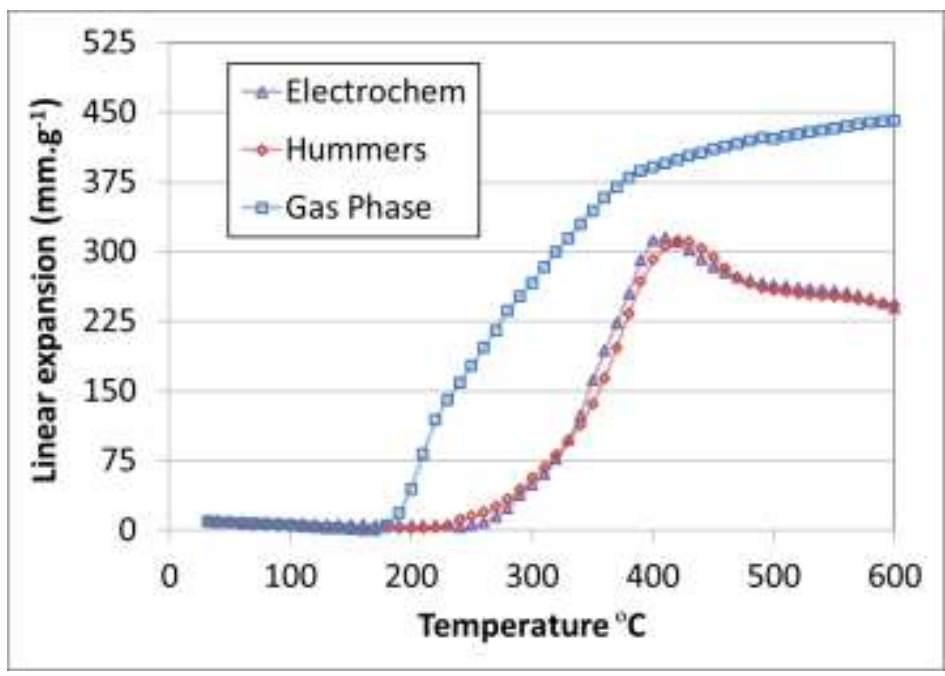

Fig. 17: Comparison of sample expansion behaviour

At first glance the GIC produced by the gas phase method seems to outperform the other two. However, this is contradicted by the specific volumes and measured surface areas of the samples, given in Table 1. Both of these indicate the gas phase method delivered the lowest expansion. 
Table 1: Sample expansion characteristics

\begin{tabular}{|c|c|c|c|}
\hline & $\begin{array}{c}\text { Specific volume } \\
\left(\mathbf{m l . g}^{-\mathbf{1}}\right)\end{array}$ & $\begin{array}{c}\text { BET surface } \\
\text { area } \\
\left(\mathbf{m}^{\mathbf{2}} \cdot \mathbf{g}^{-\mathbf{1}}\right)\end{array}$ & $\begin{array}{c}\text { TMA expansion } \\
\left(\mathbf{m l . g}^{-\mathbf{1}}\right)\end{array}$ \\
\hline Gas Phase & 45.9 & 14.1 & 8.80 \\
\hline Hummers & 68.3 & 21.7 & 4.88 \\
\hline Electrochemical & 65.8 & 18.7 & 4.84 \\
\hline
\end{tabular}

Given the explosive nature of the thermal reduction of graphite oxide and the fact that the TMA uses an applied force, the observed discrepancy may be explained. If the force could be removed it would be expected that in general the expansion of the Hummers and electrochemical would exceed that of the gas phase material. Using the size of the TMA crucible the specific volume of each sample can be calculated, as given in Table 1. These values are significantly lower than any of the measured specific volume values, indicating that in all cases full expansion was not achieved in the TMA. This makes it unlikely that the TMA measurement can provide accurate estimates of the total, unconstrained expansion. The comparative oxidative stabilities of the samples are demonstrated in Figure 18.

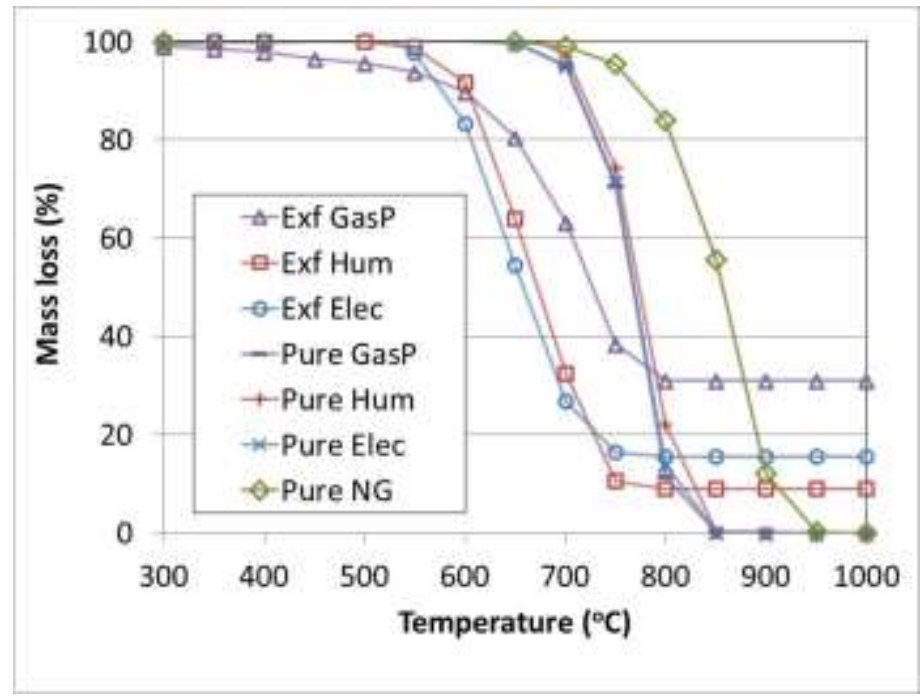

Fig. 18: Comparison of sample oxidation behaviour

In all cases the oxidative stabilities of the purified samples are significantly higher than the corresponding exfoliated samples, due to the presence of catalytic impurities. The exfoliated gas phase material exhibits a slightly lower reactivity than the other two. This is possibly related to the fact that initially the graphite is protected from 
attack, since the impurity deposits were mainly concentrated at the graphite edges. However, this material conversely has the highest impurity level of all three samples. Notably all three purified samples exhibit identical oxidation behaviour, with lower oxidative stability compared to the pure parent material. This may in part be explained by the exfoliation process which breaks down the flakes into graphite nanoplatelets, thereby increasing the exposed edge area.

In all three cases GNP's were successfully produced as indicated by their thickness of no more than a couple of hundred nanometers, combined with continuous basal surfaces in excess of several tens of micrometers. This gives the material a very high aspect ratio. However, all samples exhibit a crumpled or deformed structure. In addition, clear particle boundaries are not distinguishable, with several cross-linkages observed between particles in combination with extensive agglomeration of particles of different sizes.

None of the samples displayed the "ideal" graphite characteristics. All samples demonstrated varying levels of damage to the edge structures, for both exfoliated and purified samples. This indicates that a persistent modification of the edge microstructure has taken place. The only sample which slightly resembles the clear, straight line edges found in pure natural graphite is the modified Hummers method.

For the basal surface a mixture of intact, semi-smooth regions were found, along with regions where considerable damage was visible. The exception appears to be the electrochemical method for which it was difficult to find regions of extensive damage to the basal surface. In case of the Hummers method the damage appears to be mainly localized at the surface, with an intact underlying structure.

Unfortunately the Raman spectroscopy results were inconclusive, with all exfoliated samples yielding virtually identical traces. In all cases the so-called disorder or D-peak was practically absent, indicating that the residual damage present in the material cannot be detected by this method. This is not surprising given the fairly large size of the GNPs compared to the small spot size of the Raman laser [31]. Finally, from a yield perspective it is important to compare the mass loss curves of the samples under inert conditions, given in Figure 19. 


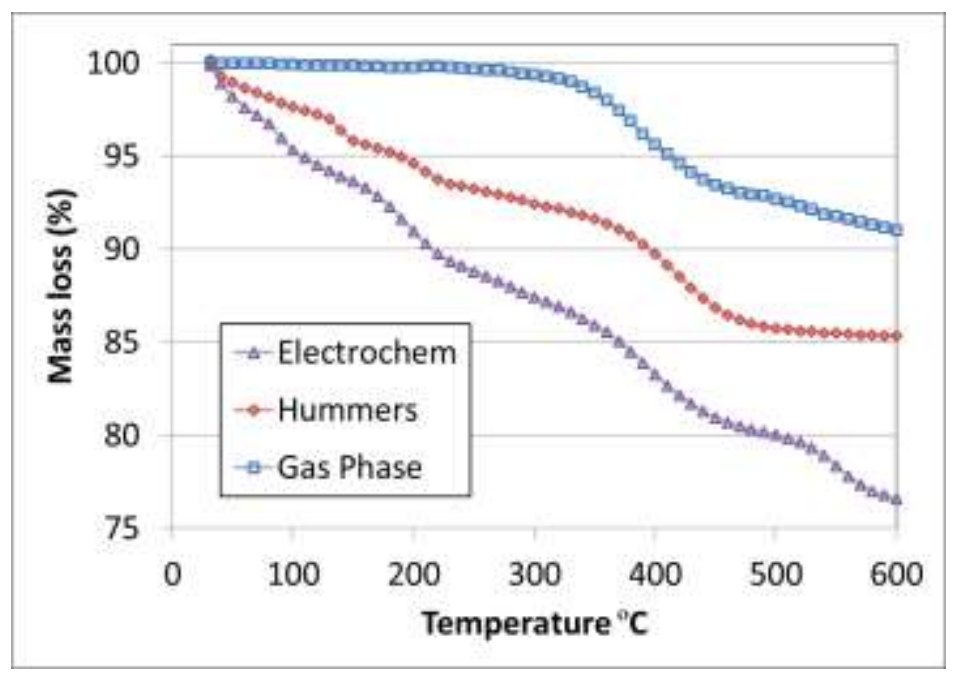

Fig. 19: Comparison of exfoliation yields

To maximize the GNP yields, materials will most likely be expanded under inert or low oxidizing conditions. The mass loss for the gas phase intercalation during the expansion process is by far the lowest at around 3\%, with the majority of the overall mass loss due to the residual impurity. For the graphite oxide based methods however, the material loss is significantly higher. In the case of the Hummers method around $15 \%$ of the starting material is lost due to gas generated by liberation of the surface bound oxygen groups and for the electrochemical method this goes up to $20 \%$.

\section{Conclusions and recommendations}

The comparative influence of different intercalation techniques on the expansion behaviour, microstructure and related characteristics of the resulting graphite nanoplatelets were investigated. The aim was to identify the method which produces exfoliated materials suitable for specific applications, in this case as an additive to enhance thermal conductivity. The three methods chosen were a gas phase intercalation of iron (III) chloride, a modified Hummers method and an electrochemical technique.

Operating parameters were varied and only the best performing samples were compared as representative. These samples do not necessary exemplify the optimal achievable result for a specific technique. The samples were mainly characterised using XRD, TMA, TGA and BET surface area. In addition the samples were extensively examined using high resolution SEM. Microwave expansion was used due to its simplicity and speed with exfoliation using a sonication probe. 
Despite the absence of strong oxidizers the electrochemical method produced a material which is very similar to the modified Hummers method in almost all respects. The XRD results show a clear distinction between the gas phase and other two methods, confirming the formation of a stage 1 intercalation compound. The other two methods both produced graphite oxide, which is confirmed by the rapid expansion behaviour and staged mass loss during thermal scanning.

The gas phase material exhibits less than 3\% mass loss during the expansion process but has a large amount of residual intercalant despite acid washing. The graphite oxide based methods result in mass loss of up to $25 \%$ in the expansion zone. For all three samples the residual impurities lead to a reduction in oxidative resistance. Once removed all samples exhibit nearly identical oxidation behaviour with an oxidative stability lower than the pure parent material.

For all three samples varying levels of damage are visible, structural deformation is wide-spread coupled with agglomeration and particle interlinking. The basal surface for the graphite oxide producing methods remains largely intact with some regions of surface damage. Remarkably the gas phase method, which is expected to only lead to insertion of atoms without disruption to the graphite, demonstrated extensive damage along the basal surface and particle edges, even when purified. This indicates that a persistent structural modification has taken place.

All three methods delivered graphite nanoplatelets with a very high aspect ratio and considerable expansion (hence effective exfoliation) as indicated by the specific volume and BET surface area. Based on these results the gas phase method performed marginally worse. The TMA analysis clearly demonstrates the difference in expansion behaviour but it is shown that this method is not accurate for determining the total unconstrained expansion. The gas phase material exhibited a more gradual expansion behaviour between 200 and $400{ }^{\circ} \mathrm{C}$, whilst the graphite oxide based methods show a very rapid expansion around $400{ }^{\circ} \mathrm{C}$. The latter is thought to be caused by the thermal desorption of a specific oxygen based surface complex.

In conclusion, the gas phase method delivers a product with significant impurities and an unexpectedly large amount of damage. This method gives a significantly better yield compared to the graphite oxide based methods but at a slightly lower effectiveness and hence product quality. The electrochemical and Hummers methods produce virtually indistinguishable products, with the Hummers method 
demonstrating marginally more 'ideal' characteristics in the final purified product. Given that high temperatures and multiple reagents requiring careful mixing are not required, the electrochemical method provides a simple, effective and potentially cheap method for large scale GNP production.

Future work will explore the relationship between the operating parameters and effectiveness of each technique. This will enable a more detailed economic analysis for choosing the optimal technique.

\section{Acknowledgements}

This work is based on research supported by the South African Research Chairs Initiative (SARChI) of the Department of Science and Technology (DST) and the National Research Foundation (NRF). Any opinion, findings and conclusions or recommendations expressed in this material are those of the authors and therefore the NRF and the DST do not accept any liability with regard thereto.

\section{References}

[1] Zhu J, Chen Z, Wang, C. Preparation and characterization of $\mathrm{CuCl} 2-\mathrm{FeCl} 3-\mathrm{H} 2 \mathrm{SO} 4-$ graphite intercalation compounds by hydrothermal synthesis. Mater Lett, 2003;57:2145-9.

[2] Asano M, Sasaki T, Abe T, Mizutani Y, Harada, T. Mass-spectrometric study of vaporization of $\mathrm{FeCl} 3$-Graphite intercalation compound. J Phys Chem Solids, 1996;57:787-90.

[3] Dresselhaus MS, Dresselhaus G. Intercalation compounds of graphite. Adv Phys, 2002;51:1:186.

[4] Zhao W, Tan PH, Liu J, Ferrari AC. Intercalation of Few-Layer Graphite Flakes with FeCl3: Raman Determination of Fermi Level, Layer by Layer Decoupling, and Stability. J Am Chem Soc, 2011;133:5941-6.

[5] Wei T, Fan Z, Luo G, Zheng C, Xie D. A rapid and efficient method to prepare exfoliated graphite by microwave irradiation. Carbon, 2008;47:313-47.

[6] Afanasov IM, Shornikova ON, Kirilenko DA, Vlasov II, Zhang L, Verbeeck J, et al. Graphite structural transformations during intercalation by HNO3 and expansion. Carbon, 2010;48:1858-65.

[7] Chung, DDL. Review Expansion of graphite. J Mater Sci, 1987;22:4190-8.

[8] Inagaki M, Tashiro R, Washino Y, Toyoda M. Expansion process of graphite via intercalation compounds with sulfuric acid. J Phys Chem Solids, 2004;65:133-7. 
[9] Łoś, S, Duclaux L, Alvarez L, Hawełek Ł, Duber S, Kempiński W. Cleavage and size reduction of graphite crystal using ultrasound radiation. Carbon, 2013;55:53-61.

[10] Du W, Jiang X, Zhu L. From graphite to graphene: direct liquid-phase exfoliation of graphite to produce single- and few layered pristine graphene. J. Mater. Chem. A, 2013;1:10592-606.

[11] Drzal LT, Fukushima H. Graphite nanoplatelets as reinforcements for polymers. Polym Prepr (Am Chem Soc, Div Polym Chem) 2001;42(2):42-3.

[12] Fukushima H, Drzal LT. A carbon nanotube alternative: Graphite nanoplatelets as reinforcements for polymers. Ann Tech Conf - Soc Plast Eng 2003:2230-4.

[13] Mhike W, Focke WW. Surface Resistivity and Mechanical Properties of

Rotationally Molded Polyethylene/Graphite Composites. J Vinyl Addit Techn, 2013;19:25870.

[14] Zhang L, Zhu J, Zhou W, Wang J, Wang, Y. Thermal and electrical conductivity enhancement of graphite nanoplatelets on form-stable polyethylene glycol / polymethyl methacrylate composite phase change materials. Energy, 2012;39:294-302

[15] Fang Z, Fan, L, Ding Q, Wang X, Yao X, Hou J, et al. Increased Thermal Conductivity of Eicosane-Based Composite Phase Change Materials in the Presence of Graphene Nanoplatelets. Energy Fuels, 2013;27:4041-7.

[16] Zeng J, Zheng S, Yu S, Zhu F, Gan J, Zhu L, et al. Preparation and thermal properties of palmitic acid/polyaniline/exfoliated graphite nanoplatelets form-stable phase change materials. Appl Energy, 2014;115:603-9.

[17] Dresselhaus MS, Dresselhaus G. Intercalation compounds of graphite. Adv Phys, 1981;30:139-326.

[18] Hummers Jr, WS, Offeman RE (1958). "Preparation of graphitic oxide." J Am Chem Soc, 1958;80:1339-55.

[19] Chung DDL. Review: Graphite. J Mater Sci. 2002;37:1475-89.

[20] Chua CK, Sofer Z, Pumera M. Graphite oxides: Effects of permanganate and chlorate oxidants on the oxygen composition. Chem Eur J, 2012;18:13453-9.

[21] Mermoux M, Chabre Y. Formation of graphite oxide. Synth Met, 1989;34:157-62.

[22] Nakajima T, Mabuchi A, Hagiwara R. A new structure model of graphite oxide. Carbon, 1988;26:357-61.

[23] Shornikova ON, Dunaev AV, Maksimove NV, Avdeev VV. Synthesis and properties of ternary GIC with iron and copper chlorides. J Phys Chem Solids, 2006;67:1193-7.

[24] Hamwi A, Senhaji A, Djurado D, Dupuis, J. Chloride-fluoride-graphite ternary compounds: Preparation and characterisation. Carbon, 1993;31:623-8. 
[25] Mizutani Y, Abe T, Asano M, Harada, T. Bi-intercalation of H2SO4 into stages 4-6 FeCl3-graphite intercalation compounds” J. Mater. Res., 1993;8:1586-95.

[26] Chen J, Yao B, Li C, Shi G. An improved Hummers method for eco-friendly synthesis of graphene oxide. Carbon, 2013;64:225-9.

[27] Poh HL, Šaněk F, Amborsi A, Zhao G, Sofer Z, Pumera M. Graphenes prepared by Staudenmaier, Hofmann and Hummers methods with consequent thermal exfoliation exhibit very different electrochemical properties. Nanoscale, 2012;4:3515-22.

[28] Kang F, Zheng Y, Wang H, Nishi Y, Inagaki M. Effect of preparation conditions on the characteristics of expanded graphite. Carbon, 2002;40:1575-81.

[29] Kalucki K, Morowski AW. Controlled Reactivity of Graphite with Iron (III) Chloride. Reac Solids, 1987;4:269-73.

[30] Nakajima T, Matsuo Y. Formation process and structure of graphite oxide, Carbon, 1994;32:469-75.

[31] Badenhorst H. Microstructure of natural graphite flakes revealed by oxidation: Limitations of XRD and Raman techniques for crystallinity estimates. Carbon, 2014;66:674-90.

[32] Abe T, Mizutani Y, Shinoda N, Ihara E, Asano M, Harada T, et al. Debye-Waller factors of FeCl3- and ICl-Graphite Intercalation Compounds. Carbon, 1995;33:1789-93.

[33] Metz W, Hohlwein D. Charakterisierung von graphit-FeCl3-verbindungen als teilweise geordnete schichtstrukturen. Carbon, 1975;13:87-96.

[34] Badenhorst H, Focke WW. Comparative analysis of graphite oxidation behaviour based on microstructure. J Nucl Mater, 2013;442:75-82.

[35] Figueiredo JL, Pereire MFR, Freitas MMA, Órfão JJM. Modification of the surface chemistry of activated carbons. Carbon, 37:1379-89.

[36] Qui Y, Guo F, Hurt R, Külaots I. Explosive thermal reduction of graphene oxide-based materials: Mechanism and safety implications. Carbon, 2014;72:215-23. 